\title{
Direct Optimization Using Gaussian Quadrature and Continuous Runge-Kutta Methods: Application to an Innovation Diffusion Model
}

\author{
Fasma Diele $^{1}$, Carmela Marangi ${ }^{1}$, and Stefania Ragni ${ }^{2}$ \\ 1 Istituto per le Applicazioni del Calcolo M. Picone, CNR, Via Amendola 122, 70126 \\ Bari, Italy \\ 2 Facoltà di Economia, Università di Bari, Via Camillo Rosalba 56, 70100 Bari, Italy
}

\begin{abstract}
In the present paper the discretization of a particular model arising in the economic field of innovation diffusion is developed. It consists of an optimal control problem governed by an ordinary differential equation. We propose a direct optimization approach characterized by an explicit, fixed step-size continuous Runge-Kutta integration for the state variable approximation. Moreover, high-order Gaussian quadrature rules are used to discretize the objective function. In this way, the optimal control problem is converted into a nonlinear programming one which is solved by means of classical algorithms.
\end{abstract}

\section{Introduction}

Dynamic optimization represents a challenging problem in several fields of applied science. From biology to engineering or economics, a wide variety of phenomena can be described in terms of optimal control problems, where a cost functional has to be optimized with respect to variables which control the system dynamics.

Numerical methods for dynamical optimization fall essentially into two classes: classical, indirect methods, relying on the maximum Pontryagin principle, with the main drawback of a lack of robustness, and so-called direct methods which attempt to find a solution through a direct optimization of the cost functional. In this paper we focus on the last type of methods and introduce a numerical scheme based on the continuous Runge-Kutta integration ([9], [11]) to solve a specific model in economics. The rationale for this choice is that an efficient and accurate approximation of the system dynamics can be obtained with a reduced number of variable evaluations. A further improvement in accuracy is achieved by adopting high-order Gauss-Legendre quadrature rules ([1]) for the functional discretization.

The approach has been tested on a specific innovation diffusion model recently proposed in [5]. The early mathematical models in this field date back to 1950 (see [7]) and represent an interesting research subject in economics, sociology, marketing as well as in applied mathematics. The problem is easily stated as the one of maximizing the profit of a monopolistic firm, due to the sale of a 
new product, by planning an advertising and price strategy. The specific model we are concerned with, is briefly described in the next section. In Section 3 we introduce the numerical approximation which combines the continuous RungeKutta integration with high-order Gauss-Legendre quadrature rules in order to discretize the constrained problem at hand. Numerical results for some values of the model parameters, shown in Section 4, validate the effectiveness of the proposed approach. Finally we draw our conclusions in Section 5.

\section{The Continuous Model}

We assume that a monopolistic firm, manufacturing a new durable good (i.e. an innovation), has to plan advertising and price strategies in order to maximize the profit due to the product sale over a finite time horizon $[0, T]$.

We suppose that the rate of adopters increase is affected by the selling price $p(t)$ according to a given price response function $q(p)$, which is usually positive and decreasing. Different price response functions have been proposed in the literature (see [6]); most of them belong to a specific class obeying the following law

$$
q(p)= \begin{cases}{[c-(1-\lambda) \alpha p]_{+}^{\frac{1}{1-\lambda}}} & \text { if } \lambda \neq 1 \\ c \exp (-\alpha p) & \text { if } \lambda=1\end{cases}
$$

with $c, \lambda, \alpha \in \mathbb{R}, \alpha>0$. Moreover, a further demand effect is represented by the amount of advertising by media $\gamma(t)$, ranging from 0 to a maximum value $\gamma_{\max }$.

Following the same approach of [5], we consider the spatial-temporal innovation diffusion accounting for an interaction among the individuals and assuming that the demand effect is related to the so-called word-of-mouth phenomenon. More precisely, the adopters are assumed to have a local influence according to a given kernel $\mathcal{K}\left(z, z^{\prime}\right)$, with $z, z^{\prime} \in \Omega$, where $\Omega \subset \mathbb{R}^{2}$ represents the geographical domain the population lives in. Under the further assumption that $\mathcal{K}\left(z, z^{\prime}\right)$ is independent of variable $z^{\prime}$, we denote

$$
\mathcal{K}=\int_{\Omega} \mathcal{K}\left(z, z^{\prime}\right) d z
$$

As a consequence, the individuals adopting the new product are uniformly distributed with respect to space variables. Then, if $\mathcal{A}(t)$ represents the adopters cumulative number at time $t$, the related evolution dynamics can be modelled as follows

$$
\frac{d \mathcal{A}}{d t}(t)=q(p(t))(\mathcal{K} \mathcal{A}(t)+\gamma(t))(1-\mathcal{A}(t)), \quad t \in[0, T]
$$

provided with condition $\mathcal{A}(0)=0$, that is no individual has adopted the innovation at initial time (for more details, see [3] and [5]).

Furthermore, we define the firm's payoff function as

$$
J(p, \gamma)=\int_{0}^{T} \exp (-r t)\left(\left(p(t)-c_{p}\right) \frac{d \mathcal{A}}{d t}(t)-c_{a} \gamma(t)\right) d t
$$


where $c_{a}, c_{p}$ and $r$ denote the per unit advertising cost, the unitary production cost and the discount factor, respectively, all assumed to be constant during the whole time horizon.

The resulting optimal control model consists of maximizing (3) with respect to control parameters represented by price and advertising, governed by state equation (2). More precisely, defining $\mathcal{F}$ as the space of all the functions mapping $[0, T]$ into $\mathbb{R}$ and setting

$$
\mathcal{U}=\left\{(u, v) \in \mathcal{F}^{2} \mid u, v \text { are piecewise continuous, } 0 \leq u(t), 0 \leq v(t) \leq \gamma_{\max }\right\},
$$

the problem reduces to

$$
\max _{p, \gamma \in \mathcal{U}} J(p, \gamma)
$$

constrained by equation (2) with condition $\mathcal{A}(0)=0$. For a theoretical analysis of the model see [5]. Moreover, in 4] a preliminary numerical solution of the problem is given accounting for spatial effects of the new product diffusion in the market; in that case the optimal control model is governed by a partial differential equation.

\section{Numerical Approximation}

Our main interest consists of performing the numerical discretization of the previous continuous model based on the use of continuous extensions of RungeKutta methods combined with Gaussian quadrature rules.

Let us introduce a mesh $0=t_{0}<t_{1}<\ldots<t_{N}=T$ of the given time interval $[0, T]$ with constant step length $h$, then $t_{i}=i h$ for each $i=0, \ldots N$. Moreover, setting

$$
\psi(\mathcal{A}(t), p(t), \gamma(t), t)=q(p(t))(\mathcal{K} \mathcal{A}(t)+\gamma(t))(1-\mathcal{A}(t)),
$$

we define

$$
g(p(t), \gamma(t), \mathcal{A}(t), t)=\exp (-r t)\left(\left(p(t)-c_{p}\right) \psi(\mathcal{A}(t), p(t), \gamma(t), t)-c_{a} \gamma(t)\right) .
$$

Hence, the integral in (3) can be written as

$$
\begin{aligned}
& \int_{0}^{T} g(p(t), \gamma(t), \mathcal{A}(t), t) d t=\sum_{i=0}^{N-1} \int_{t_{i}}^{t_{i+1}} g(p(t), \gamma(t), \mathcal{A}(t), t) d t \\
& =\sum_{i=0}^{N-1} \frac{h}{2} \int_{-1}^{1} g\left(p\left(t_{i}+h \frac{s+1}{2}\right), \gamma\left(t_{i}+h \frac{s+1}{2}\right), \mathcal{A}\left(t_{i}+h \frac{s+1}{2}\right), t_{i}+h \frac{s+1}{2}\right) d s .
\end{aligned}
$$

We approximate every integral by means of the well-known Gauss-Legendre rules; thus, the right-hand term in (5) is discretized as

$$
\frac{h}{2} \sum_{i=0}^{N-1} \sum_{l=0}^{L} w_{l} g\left(p\left(\tau_{i, l}\right), \gamma\left(\tau_{i, l}\right), \mathcal{A}\left(\tau_{i, l}\right), \tau_{i, l}\right)
$$


where

$$
\tau_{i, l}=t_{i}+h \frac{s_{l}+1}{2}, \quad i=0, \ldots, N-1, l=0, \ldots, L
$$

and $s_{l}, w_{l}$ represent the knots (i.e. the zeros of Legendre polynomial of order $L+1$ ) and the coefficients, respectively, of the chosen quadrature scheme (see 1]). Notice that the quantities involved in the previous approximation are the values of both the controls $p, \gamma$ and the state variables $\mathcal{A}$ as sampled at each instant $\tau_{i, l}$.

If we assume to approximate control parameters in the class of piecewise linear functions

$$
\mathcal{V}=\left\{v \in \mathcal{F}|v|_{\left[t_{i}, t_{i+1}\right]} \text { is linear }\right\}
$$

we have that

$$
\begin{aligned}
& p\left(\tau_{i, l}\right)=\rho_{l} p\left(t_{i}\right)+\xi_{l} p\left(t_{i+1}\right), \\
& \gamma\left(\tau_{i, l}\right)=\rho_{l} \gamma\left(t_{i}\right)+\xi_{l} \gamma\left(t_{i+1}\right)
\end{aligned}
$$

with $\rho_{l}=\frac{1-s_{l}}{2}$ and $\xi_{l}=1-\rho_{l}$ for every $l=0, \ldots, L$.

Moreover, in order to evaluate $\mathcal{A}\left(\tau_{i, l}\right)$ 's, the state equation is discretized by applying continuous extensions of Runge-Kutta methods. We consider an explicit $m$-stage scheme specified by the Butcher array

$$
\begin{array}{l|l}
\mathbf{c} & A \\
\hline & \mathbf{b}^{T}
\end{array}
$$

and its continuous extension characterized by coefficients $\widetilde{b}_{j}(\theta)(j=1, \ldots, m)$ which are suitable polynomials in $\theta \in[0,1]$ obtained as in [11]. The resulting scheme for the state system is the following

$$
\begin{aligned}
& \mathcal{A}\left(\tau_{i, l}\right)=\mathcal{A}\left(t_{i}\right)+h \sum_{j=1}^{m} \widetilde{b}_{j}\left(\xi_{l}\right) \psi\left(\widetilde{\mathcal{A}}_{j}, p\left(t_{i}+h c_{j}\right), \gamma\left(t_{i}+h c_{j}\right), t_{i}+h c_{j}\right), \\
& \mathcal{A}\left(t_{i+1}\right)=\mathcal{A}\left(t_{i}\right)+h \sum_{j=1}^{m} \widetilde{b}_{j}(1) \psi\left(\widetilde{\mathcal{A}}_{j}, p\left(t_{i}+h c_{j}\right), \gamma\left(t_{i}+h c_{j}\right), t_{i}+h c_{j}\right), \\
& \widetilde{\mathcal{A}}_{j}=\mathcal{A}\left(t_{i}\right)+h \sum_{v=1}^{j-1} a_{j, v} \psi\left(\widetilde{\mathcal{A}}_{v}, p\left(t_{i}+h c_{v}\right), \gamma\left(t_{i}+h c_{v}\right), t_{i}+h c_{v}\right), j=1, \ldots, m
\end{aligned}
$$

starting from $\mathcal{A}\left(t_{0}\right)=0$.

Note that condition $0 \leq \xi_{l} \leq 1$ holds for each $l=0, \ldots L$. Furthermore, in every interval $\left(t_{i}, t_{i+1}\right)$ we should add extra control variables at each inner time step $\sigma_{i, j}:=t_{i}+h c_{j}$ since, in general, $\sigma_{i, j} \neq \tau_{i, l}$. However, additional controls might be avoided simply by choosing Runge-Kutta scheme in such a way that $c_{1}=0, c_{m}=1$ and every inner $c_{j}$ is equal to $\xi_{l}$ for some $l=0, \ldots, L$.

Finally, let us consider the following vector

$$
\mathbf{u}=\left(p\left(t_{0}\right), p\left(t_{1}\right), \ldots, p\left(t_{N}\right), \gamma\left(t_{0}\right), \gamma\left(t_{1}\right), \ldots, \gamma\left(t_{N}\right)\right)^{T} \in \mathbb{R}^{2(N+1)} .
$$


In this notation, the sum in (6) can be defined as

$$
\widetilde{J}(\mathbf{u})=\frac{h}{2} \sum_{i=0}^{N-1} \sum_{l=0}^{L} w_{l} g\left(\rho_{l} u_{i+1}+\xi_{l} u_{i+2}, \rho_{l} u_{N+2+i}+\xi_{l} u_{N+3+i}, \mathcal{A}\left(\tau_{i, l}\right), \tau_{i, l}\right)
$$

where each $u_{j}$ denotes the $j$-th entry of vector $\mathbf{u}$ and the values $\mathcal{A}\left(\tau_{i, l}\right)$, depending on $\mathbf{u}$ itself, are computed by recursive formula (7). In this way, the numerical approximation of the optimal control model described in Section 2 yields a solution to the nonlinear programming problem

PROBLEM 1 Maximize $\widetilde{J}(\mathbf{u})$ defined in (8) and constrained by

$$
\begin{array}{ll}
0 \leq u_{i}, & i=1, \ldots, 2(N+1), \\
u_{N+1+i} \leq \gamma_{\max }, & i=1, \ldots, N+1 .
\end{array}
$$

We note the proposed numerical discretization follows an idea similar to the one provided by authors in [2].

\section{Numerical Tests}

In order to test the effectiveness of the numerical procedure described in the previous section, we use the Gauss-Legendre rule with 2 knots (i.e. $L=1$ ); furthermore, the chosen Runge-Kutta scheme is the continuous extension of the 2-stage improved Euler method (see [8]) where

$$
b_{1}(\theta)=-\frac{1}{2} \theta^{2}+\theta \quad \text { and } \quad b_{2}(\theta)=\frac{1}{2} \theta^{2} .
$$

Discrete solutions have been achieved solving nonlinear programming Problem 1 in Matlab environment by means of fmincon routine.

In our numerical examples, the price response function is defined in correspondence with $\lambda=c=1$ in (1). We set the discount factor $r=0$, since in this case the theoretical solution of the continuous model is known; in particular, in [5] it has been proven that optimal price is characterized by a concave behaviour, while advertising strategy is a bang-bang function.

In Figures 1 and 2, we plot the approximate time evolution of price and advertising strategies as well as adopters cumulative number obtained for different values of parameters specified in each caption. As it is shown in figures, numerical results are in agreement with theoretical ones. We remark that the case in Figure 2 shows an evident bang-bang behaviour which cannot be catched by classical gradient algorithms. A further improvement could be obtained by exploiting the advantages of global stochastic optimization approaches.

\section{Conclusions}

A numerical approximation has been provided in order to solve a specific economic model which describes the spread of a new product in a market and deals 

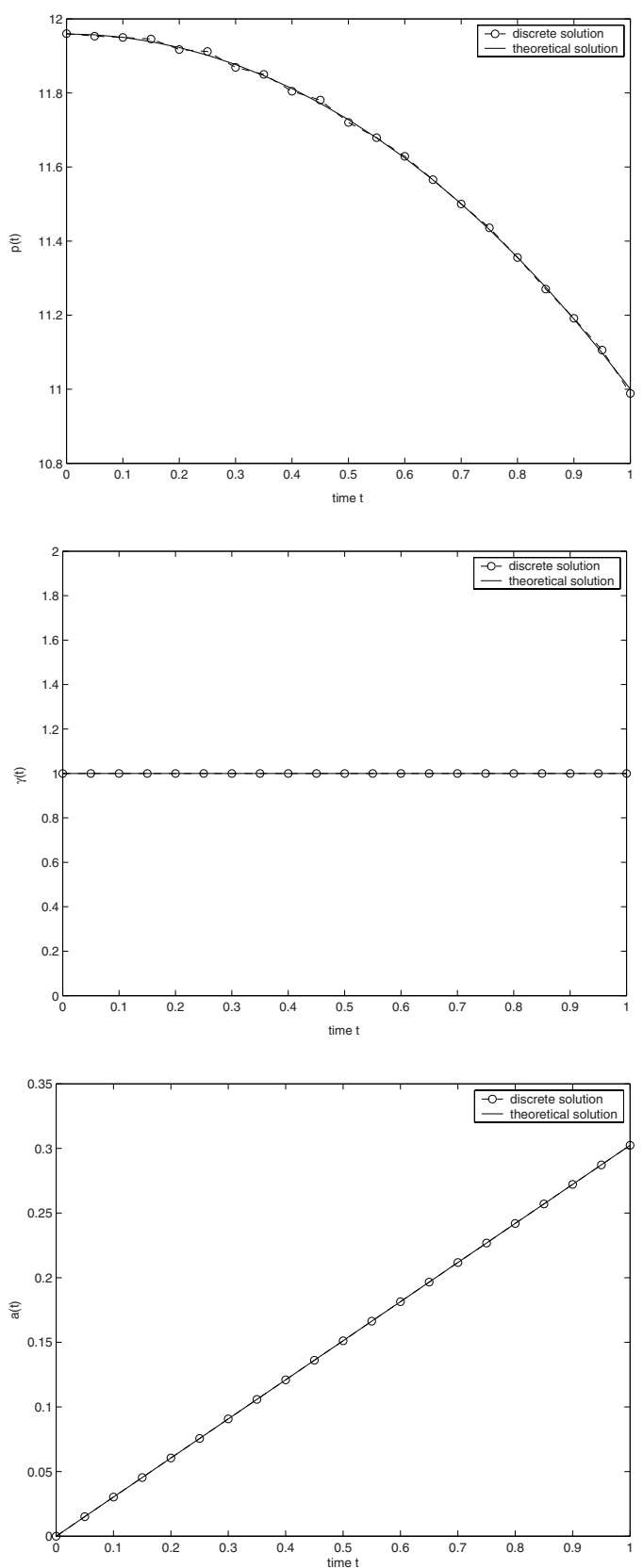

Fig. 1. Optimal price (on the top), advertising (in the middle) and adopters cumulative number (on the bottom) in correspondence with $T=1, \gamma_{\max }=1, \mathcal{K}=1, \alpha=0.1$, $c_{a}=1$ and $c_{p}=1$. Time step length is $h=0.05$. 

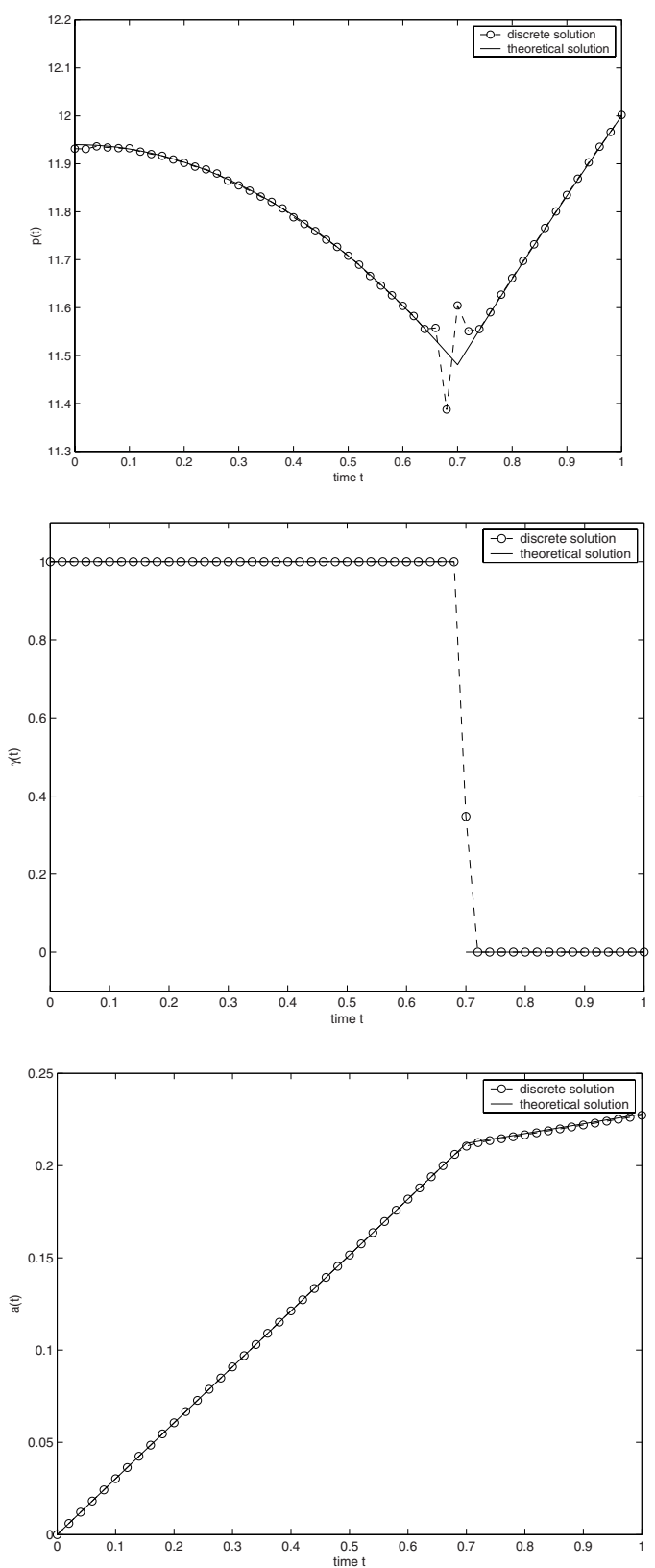

Fig. 2. Optimal price (on the top), advertising (in the middle) and adopters cumulative number (on the bottom) in correspondence with $T=1, \gamma_{\max }=1, \mathcal{K}=1, \alpha=0.1$, $c_{a}=2.5$ and $c_{p}=2$. Time step length is $h=0.02$. 
with the choice of price and advertising strategies maximizing the profit coming from its sale. The discrete procedure reduces a given optimal control problem governed by ordinary differential equations to a nonlinear programming one. More precisely, the discrete scheme is characterized by a direct optimization procedure obtained by using Gauss-Legendre quadrature rules in order to approximate the integral optimizing functional and continuous Runge-Kutta methods for the state variables differential constraints. The main advantage of this approach is that it allows the reduction of nodal variable values necessary for the chosen discretization while retaining a great accuracy in the solution. Finally, the numerical results confirm the effectiveness of the proposed algorithm.

\section{References}

1. Davis, P.J., Rabinowitz, P.: Methods of numerical integration, 2nd Ed., Academic, New York (1984).

2. Diele, F., Marangi, C., Ragni, S.: Numerical methods based on Gaussian quadrature and continuous Runge-Kutta integration for optimal control problems. IAC Thecnical Report N.33 (2/2004).

3. De Cesare, L., Di Liddo, A.: A Bolza optimal control problem for innovation diffusion. Dynamic Systems and Appl., 9 (2000) 269-280.

4. De Cesare, L., Di Liddo, A., Ragni, S.: Numerical solutions to some optimal control problems arising from innovation diffusion. Computational Economics, 22 (2003) $173-186$.

5. De Cesare, L., Di Liddo, A., Ragni, S.: On the shape of optimal price and advertising strategies arising in innovation diffusion. Accepted for publication in Dynamic Systems and Appl.

6. Kalish, S., Lilien, G. L.: Optimal price subsidy for accelerating the diffusion of innovation. Marketing Science, 2 (1983) 407-420.

7. Kendall, D. G.: Discussion on Bartlett, M. S., Measles, periodicuty and community size'. J. Roy. Stat. Soc. Ser. A, 120 (1957) 48-70.

8. Lambert, J. D.: Numerical methods for ordinary differential systems. Wiley \& Sons, Chichester (1997).

9. Owren, B., Zennaro, M.: Derivation of efficient, continuous, explicit Runge-Kutta methods. SIAM J. Sci. Stat. Comput., Vol. 13, No. 6 (1992) 1488-1501.

10. Schwartz, A.: Theory and implementation of numerical methods based on RungeKutta integration for solving optimal control problems. PhD Thesis, U.C. Berkeley (1996).

11. Zennaro, M.: Natural continuous extensions of Runge-Kutta methods. Mathematics of Computation, Vol. 46, No. 173 (1986) 119-133. 